\title{
HYPERBOLICITY OF A COMPLEX MANIFOLD AND OTHER EQUIVALENT PROPERTIES
}

\author{
KYONG T. HAHN ${ }^{1}$ AND KANG T. KIM
}

\begin{abstract}
ABSTRaCr. Defining the notions of Schottky, Landau and Picard properties on a plane domain, the first author [3] proved that a domain in $\mathbf{C}$ having any of these properties is equivalent to the hyperbolicity of the domain.

In this paper the authors extend these notions to higher-dimensional case and obtain other various equivalent conditions for the hyperbolicity of a complex manifold.
\end{abstract}

1. Introduction. Let $M$ be a connected complex manifold of dimension $m$ with hermitian metric $h_{M}$. Let $\rho_{M}$ denote the distance function associated with $h_{M}$. Following S. Kobayashi [6], we define $M$ to be (Kobayashi) hyperbolic if the Kobayashi pseudometric $k_{M}$ is a metric. In [8], H. Royden has constructed the infinitesimal form of the Kobayashi pseudometric

$$
K_{M}(p, \xi)=\inf \left\{|v|: \exists f \in \mathcal{H}(\Delta, M) \ni f(0)=p, f^{\prime}(0) v=\xi\right\}
$$

where $(p, \xi) \in T(M)$, the tangent bundle of $M$, and $\mathcal{H}(\Delta, M)$ denotes the class of all holomorphic maps $f$ of the open disc $\Delta$ into $M$, and he has shown that if $M$ is hyperbolic then $k_{M}$ is the integrated form of $K_{M}$. The notion of hyperbolicity of $M$ can also be defined in terms of $K_{M}$ : A complex manifold $M$ is hyperbolic if for each $p \in M$ there exists a coordinate neighborhood $U$ of $p$ and a positive constant $c$ such that for $(q, \eta) \in T(U) \simeq U \times \mathbf{C}^{m}$

$$
K_{M}(q, \eta) \geqslant c\|\eta\|
$$

where $\|\cdot\|$ denotes the euclidean distance. This definition of hyperbolicity coincides with that of Kobayashi (see [8]). In the case of a hermitian manifold, the above condition is equivalent to the condition that for each $p \in M$ there is a neighborhood $U$ of $p$ and a positive constant $c$ such that

$$
K_{M}(q, \eta) \geqslant c h_{M}(q, \eta)
$$

for all $(q, \eta) \in T(U)$.

Definition 1. A complex manifold $M$ is said to satisfy the Schottky property if for each $p \in M$, for each relatively compact open set $W$ in a coordinate neighborhood of $p$ and an $r \in(0,1)$, there exists a positive constant $S=S(W, r)$ such that every

Received by the editors June 23, 1983.

1980 Mathematics Subject Classification. Primary 32H20, 32H25; Secondary 32H15.

Key words and phrases. Schottky property, Landau property, Picard property, Bloch mapping, Kobayashi metric and hyperbolic manifold.

'Research partially supported by NSF Grant MCS 80-02915 at Pennsylvania State University. 
holomorphic map $f \in \mathcal{H}(\Delta, M)$ with $f(0) \in W$ satisfies

$$
\rho_{M}(p, f(z)) \leqslant S \text { for }|z| \leqslant r .
$$

$M$ satisfies the Landau property if for each $p \in M$ and each relatively compact open set $W$ in a coordinate neighborhood of $p$, there exists a positive constant $R=R(W)$ such that

$$
\sup \left\{h_{M}\left(f(0), f^{\prime}(0)\right): f \in \mathcal{H}(\Delta, M) \text { with } f(0) \in W\right\} \leqslant R .
$$

Then we can prove the following.

THEOREM 1. The following statements are equivalent on any hermitian manifold $M$.

(a) $M$ is hyperbolic.

(b) M satisfies the Schottky property.

(c) M satisfies the Landau property.

Definition 2. A map $f \in \mathscr{H}(\Delta, M)$ is said to be Bloch (see [4]) if

$$
\sup \left\{Q_{f}(z): z \in \Delta\right\}<\infty,
$$

where

$$
Q_{f}(z)=\left(1-|z|^{2}\right) h_{M}\left(f(z), f^{\prime}(z)\right) .
$$

We remark that the nonnegative function $Q_{f}$ is invariant under the group $\operatorname{Aut}(\Delta)$ of holomorphic automorphisms of $\Delta$ in the sense that for all $\varphi \in \operatorname{Aut}(\Delta)$

$$
Q_{f \circ \varphi}(z)=Q_{f}(\varphi(z)), \quad z \in \Delta .
$$

It is an easy consequence of the definition of Kobayashi metric that if (5a) holds for all $f \in \mathscr{H}(\Delta, M)$, then $M$ is hyperbolic. The converse is, however, not true in general (see lemma in $\S 3$ ). If $M$ is compact, we obtain the following characterization of the hyperbolicity of $M$.

THEOREM 2. Let $M$ be a connected compact hermitian manifold. The following statements are equivalent.

(a) $M$ is hyperbolic.

(b) $\sup \left\{h_{M}\left(f(0), f^{\prime}(0)\right): f \in \mathcal{H}(\Delta, M)\right\}<\infty$.

(c) $\sup \left\{Q_{f}(z): z \in \Delta\right\}<\infty$ for all $f \in \mathcal{H}(\Delta, M)$, i.e., every $f \in \mathcal{H}(\Delta, M)$ is Bloch.

(d) $M$ has the Picard property, i.e., there is no nonconstant holomorphic map $f$ : C $\rightarrow$ M.

Unlike in the one complex-dimensional case [3], the notion of the Picard property is a weaker notion than the Schottky property, the Landau property or the hyperbolicity for a noncompact manifold [6].

We note that the notion of the Picard property used in [3] and here, somewhat reluctantly, coincides with the term previously used by S. Kobayashi [6] and others.

Finally, we acknowledge our indebtedness to S. Krantz ard also to the referee for their helpful comments on the paper. In particular, the example given in the proof of the second half of the lemma was suggested by the referee. This example is much simpler than the one originally given by the authors. 
2. Proof of Theorem 1. It will be proved through the following implications: (a) $\Leftrightarrow($ b) , (a) $\Rightarrow$ (c) $\Rightarrow$ (a).

To prove (a) $\Rightarrow$ (b), let $p \in M$ and $U$ be its local coordinate neighborhood. Let $W$ be a relatively compact neighborhood of $p$ in $U$. Since $k_{M}$ is a metric, it induces the standard topology in $M$ [1]. Therefore, we may define $W=\left\{q: k_{M}(p, q)<\rho_{0}\right\}$ for some $\rho_{0}>0$ and $U=\left\{q: k_{M}(p, q)<\rho_{0}+\rho_{1}\right\}$, where $\rho_{1}=\tan h^{-1} r$, for any given $r \in(0,1)$. If $f \in \mathcal{H}(\Delta, M)$ satisfies $f(0) \in W$, then $k_{M}(p, f(0))<\rho_{0}$ and $k_{M}(f(0), f(z)) \leqslant k_{\Delta}(0, z) \leqslant \rho_{\Delta}(0, r)=\tan h^{-1} r=\rho_{1}$ for $|z| \leqslant r$. Therefore, it follows from the triangle inequality that $k_{M}(p, f(z)) \leqslant \rho_{0}+\rho_{1}$ whenever $|z| \leqslant r$. Since $k_{M}$ is a metric, $k_{M} \geqslant c \rho_{M}$ for some $c>0$ which proves (b) with $S=\left(\rho_{0}+\rho_{1}\right) / c$.

For the proof of (b) $\Rightarrow$ (a), we follow the method of [5]: Let $p$ and $q$ be two distinct points of $M$. We may take a coordinate neighborhood $U$ of $p$ which does not contain $q$. Let $\varphi: U \rightarrow B=\left\{w \in \mathbf{C}^{m}:\|w\|<1\right\}$ be a biholomorphic map with $\varphi(p)=0, \varphi(U)=B$. Without loss of generality, we may assume that $\varphi(W)=\{w \in$ $\left.\mathbf{C}^{m}:\|w\|<\rho\right\}=B_{\rho}$ for some $\rho \in(0,1)$ and $\varphi(V) \subset B, V=\left\{m \in M: \rho_{M}(p, M)<\right.$ $S$ ). Let $r \in(0,1)$ be given. If $|z|<r / 2$, then there exists a constant $c>0$ such that $\tan h^{-1}|z| \geqslant c \tan h^{-1}|z| / r$ or, equivalently, $\rho_{\Delta}(0, z) \geqslant c \rho_{\Delta_{r}}(0, z)$. Let $\alpha=\{p=$ $\left.p_{0}, p_{1}, \ldots, p_{l}=q ; a_{1}, \ldots, a_{l} ; f_{1}, \ldots, f_{l}\right\}$ be any chain connecting $p$ and $q$, used in the construction of the Kobayashi metric $k_{M}(p, q)$ [6]. Since $\varphi(q)$ lies outside $B$, there must be an index, say $l_{0}, 0 \leqslant l_{0}<l$, such that $\varphi\left(p_{0}\right), \varphi\left(p_{1}\right), \ldots, \varphi\left(p_{l_{0}-1}\right) \in B_{\rho}$ and $\varphi\left(p_{l_{0}}\right) \notin B_{\rho}$. By the homogeneity of $\Delta$, we may assume that $a_{1}, \ldots, a_{l_{0}}$ lie in $\Delta_{r / 2}$. Then

$$
\begin{gathered}
|\alpha| \geqslant \sum_{i=1}^{l_{0}} \rho_{\Delta}\left(0, a_{i}\right) \geqslant c \sum_{i=1}^{l_{0}} \rho_{\Delta_{r}}\left(0, a_{i}\right) \geqslant c \sum_{i=1}^{l_{0}} k_{B}\left(\varphi\left(p_{i-1}\right), \varphi\left(p_{i}\right)\right) \\
\geqslant c k_{B}\left(0, \varphi\left(p_{l_{0}}\right)\right)=c \tan h^{-1}\left\|\varphi\left(p_{l_{0}}\right)\right\| \geqslant c \tan h^{-1} \rho \equiv c^{\prime} .
\end{gathered}
$$

Thus, $k_{M}(p, q) \geqslant c^{\prime}>0$. Here we used the fact that on $B$ the Kobayashi metric agrees with the standard Kähler metric. It is given by

$$
k_{B}(0, z)=\frac{1}{2} \log \frac{1+\|z\|}{1-\|z\|}=\tan h^{-1}\|z\| \quad \text { for } z \in B .
$$

See [4].

(a) $\Rightarrow$ (c). Suppose that $M$ fails to satisfy the Landau property. Then there exist a point $p_{0}$ in an open neighborhood $W$ which is relatively compact in a local coordinate neighborhood $U$ of $p_{0}$ and a sequence $f_{k} \in \mathcal{H}(\Delta, M)$ such that $f_{k}(0) \in W$ and $h_{M}\left(f_{R}(0), f_{k}^{\prime}(0)\right) \rightarrow \infty$ as $k \rightarrow \infty$. We claim that if $M$ is hyperbolic then there exists an $r \in(0,1)$ such that $\left\{f_{k}\right\}$ contains a subsequence which converges uniformly to a holomorphic map $f: \Delta_{r} \rightarrow M$. First we observe that $\bar{W} \cap(M \backslash U)=\varnothing$ where $\bar{W}$ is compact and $M \backslash U$ is closed. Therefore, there exists a number $\rho^{\prime}>0$ such that

$$
Q=\left\{p \in M: k_{M}(\bar{W}, p)<\rho^{\prime}\right\} \subset \subset U .
$$

Since $k_{M}\left(f_{k}(0), f_{k}(z)\right) \leqslant \rho_{\Delta}(0, z)=\tan h^{-1}|z|$ for all $f_{k} \in \mathcal{H}(\Delta, M)$ with $f_{k}(0) \in W$, $f_{k}(z) \in Q$ whenever $|z| \leqslant r^{\prime}=\tan h \rho^{\prime}$. Since $Q$ is relatively compact, it is bounded in $\mathbf{C}^{m}$. Therefore, by Montel's theorem there is a subsequence of $\left\{f_{k}\right\}$ which 
converges uniformly on $\Delta_{r}$ to a holomorphic map $f: \Delta_{r} \rightarrow M$ for $r<r^{\prime}$. Denote again this convergent subsequence by $\left\{f_{k}\right\}$. By Weierstrass' theorem, $f_{k}^{\prime}(z)$ converges to $f^{\prime}(z)$ uniformly on $\Delta_{r}$. In particular, $f_{k}^{\prime}(0)$ converges to $f^{\prime}(0)$. Thus, $h_{M}\left(f_{k}(0), f_{k}^{\prime}(0)\right) \rightarrow h_{M}\left(f(0), f^{\prime}(0)\right)<\infty$ as $k \rightarrow \infty$, which is a contradiction.

(c) $\Rightarrow$ (a). Given $(q, \xi) \in T(W)$ and $v \in \mathbf{C}$, let $f \in \mathcal{H}(\Delta, M)$ satisfy $f(0)=q$ and $f^{\prime}(0) v=\xi$. Then by $(4)$ of $\S 1$

$$
R|v| \geqslant h_{M}\left(f(0), f^{\prime}(0)\right)|v|=h_{M}\left(f(0), f^{\prime}(0) v\right)=h_{M}(q, \xi)
$$

or

$$
|v| \geqslant \frac{1}{R} h_{M}(q, \xi),
$$

where $R$ is the upper bound given in (4) of $\S 1$. Thus,

$$
K_{M}(q, \xi) \geqslant c h_{M}(q, \xi)
$$

$c=1 / R$, as desired.

3. Proof of Theorem 2. By a result of R. Brody [2], (a) $\Leftrightarrow$ (d) holds on a connected compact manifold. Therefore, it is enough to prove the implications (a) $\Rightarrow(b) \Rightarrow$ (c) $\Rightarrow$ (a). First we prove the following

Lemma. A complex manifold $M$ is hyperbolic if $\sup \left\{Q_{f}(z): z \in \Delta\right\}<\infty$ for all $f \in \mathcal{H}(\Delta, M)$. The converse is not true in general.

Proof. The above hypothesis implies that there exists a constant $Q>0$ such that for all $f \in \mathcal{H}(\Delta, M)$

$$
h_{M}\left(f(z), f^{\prime}(z) v\right) \leqslant Q K_{\Delta}(z, v) \quad(z \in \Delta, v \in \mathbf{C}) .
$$

Let $\tilde{\rho}_{M}$ be the distance function associated with the differential metric $\tilde{h}_{M}=Q^{-1} h_{M}$. By integrating both sides of (1) along the geodesic curve between two points $z$ and $w$ in $\Delta$ and using the definition of integrated distance $\tilde{\rho}_{M}$, we have

$$
\tilde{\rho}_{M}(f(z), f(w)) \leqslant k_{\Delta}(z, w)
$$

for all $f \in \mathcal{H}(\Delta, M)$. Since $k_{M}$ is the largest among those pseudometrics which satisfy (2), $\tilde{\rho}_{M} \leqslant k_{M}$ (see [6]). Thus, $M$ is hyperbolic.

To show the second half, let $M$ be the right half-plane of $\mathbf{C}$ and $h_{M}$ the euclidean metric. Then $M$ is clearly hyperbolic. Set

$$
f(z)=(1+z) /(1-z) \text { and } z_{n}=1-1 / n \in \Delta .
$$

Then $f \in \mathcal{H}(\Delta, M)$ and

$$
Q_{f}\left(z_{n}\right)=\left(1-\left|z_{n}\right|^{2}\right)\left|f^{\prime}\left(z_{n}\right)\right|=2(2 n-1) \rightarrow \infty
$$

as $n \rightarrow \infty$. This completes the lemma.

From this lemma, (c) $\Rightarrow$ (a) trivially follows. To prove (a) $\Rightarrow$ (b), let $M$ be hyperbolic. By (c) of Theorem 1 , for each $p \in M$ there exists a relatively compact open neighborhood $W_{p}$ of $p$ such that

$$
\sup \left\{\left\|f^{\prime}(0)\right\|: f \in \mathcal{K}(\Delta, M), f(0) \in W_{p}\right\}<\infty .
$$


Since $M$ is compact, it can be covered by a finite number of such $W_{p}$ 's. Thus, (b) follows.

(b) $\Rightarrow$ (c). Suppose (c) fails to hold. Then there exist sequences $\left\{z_{n}\right\}$ in $\Delta$ and $\left\{f_{n}\right\}$ in $\mathcal{H}(\Delta, M)$ such that $Q_{f_{n}}\left(z_{n}\right)>n$ for all $n$. Since $\Delta$ is homogeneous, for each $z_{n}$ there exists $\varphi_{n} \in \operatorname{Aut}(\Delta)$ such that $\varphi_{n}(0)=z_{n}$. Therefore, by the invariant property of $Q_{f}(\operatorname{see}(6)$ of $\S 1)$,

$$
Q_{f_{n}}\left(z_{n}\right)=Q_{f_{n}}\left(\varphi_{n}(0)\right)=Q_{g_{n}}(0)=h_{M}\left(g_{n}(0), g_{n}^{\prime}(0)\right),
$$

where $g_{n}=f_{n} \circ \varphi_{n}$. Since $h_{M}$ is hermitian, it follows from the compactness of $M$, condition (b) and (5) that $Q_{f_{n}}\left(z_{n}\right)$ is bounded for all $n$, which is a contradiction.

\section{REFERENCES}

1. T. J. Barth, The Kobayashi distance induces the standard topology, Proc. Amer. Math. Soc. 35 (2972), 439-441. MR 46 \#5668.

2. R. Brody, Compact manifolds and hyperbolicity, Bull. Amer. Math. Soc. 235 (1978), 213-219.

3. K. T. Hahn, Equivalence of the classical theorems of Schottky, Landau, Picard and hyperbolicity, Proc. Amer. Math. Soc. 89 (1983), 628-632.

4. __ Asymptotic behavior of normal mappings of several complex variables, Canad. J. Math (to appear).

5. P. Kiernan, On the relation between taut, tight and hyperbolic manifolds, Bull. Amer. Math. Soc. $\mathbf{7 6}$ (1970), 49-51. MR 40 \#5896.

6. S. Kobayashi, Hyperbolic manifolds and holomorphic mappings, Pure and Appl. Math., vol. 2, Dekker, New York, 1970. MR 43 \#3503.

7. M. Kwack, Some classical theorems for holomorphic mappings into hyperbolic manifolds, Proc. Sympos. Pure Math., vol. 27, Amer. Math. Soc., Providence, R. I., 1975, pp. 99-104.

8. H. Royden, Remarks on the Kobayashi metric (Proc. Maryland Conf. Several Complex Variables). Lecture Notes in Math., vol. 185, Springer-Verlag, Berlin, 1971, pp. 125-137. MR 46 \#826.

Department of Mathematics, Pennsylvania State University, University Park, Pennsylvania 16802

Department of Mathematics, University of California, Los Angeles, California 90024 